\title{
Enhanced Critical Size Defect Repair in Rabbit Mandible by Electrospun Gelatin/ $\beta$-TCP Composite Nanofibrous Membranes
}

\author{
Mingming Xu, ${ }^{1}$ Xuehui Zhang, ${ }^{2}$ Song Meng, ${ }^{1}$ Xiaohan Dai, \\ Bing Han, ${ }^{3}$ and Xuliang Deng ${ }^{1,4,5}$ \\ ${ }^{1}$ Department of Geriatric Dentistry, Peking University School and Hospital of Stomatology, Beijing 100081, China \\ ${ }^{2}$ Department of Dental Materials, Peking University School and Hospital of Stomatology, Beijing 100081, China \\ ${ }^{3}$ Department of Orthodontics, Peking University School and Hospital of Stomatology, Beijing 100081, China \\ ${ }^{4}$ National Engineering Laboratory for Digital and Material Technology of Stomatology, Beijing 100081, China \\ ${ }^{5}$ Beijing Laboratory of Biomedical Materials, Peking University School and Hospital of Stomatology, Beijing 100081, China
}

Correspondence should be addressed to Bing Han; orthohanks@sina.com and Xuliang Deng; kqdengxuliang@bjmu.edu.cn

Received 10 October 2014; Accepted 15 December 2014

Academic Editor: Krasimir Vasilev

Copyright (c) 2015 Mingming Xu et al. This is an open access article distributed under the Creative Commons Attribution License, which permits unrestricted use, distribution, and reproduction in any medium, provided the original work is properly cited.

\begin{abstract}
The design and fabrication of biodegradable barrier membranes with satisfactory structure and composition remain a considerable challenge for periodontal tissue regeneration. We have developed a biomimetic nanofibrous membrane made from a composite of gelatin and $\beta$-tricalcium phosphate $(\beta$-TCP). We previously confirmed the in vitro biological performance of the membrane material, but the efficacy of the membranes in promoting bone repair in situ has not yet been examined. Gelatin/ $\beta$-TCP composite nanofibers were fabricated by incorporation of $20 \mathrm{wt} . \% \beta$-TCP nanoparticles into electrospun gelatin nanofibers. Electron microscopy showed that the composite membranes presented a nonwoven structure with an interconnected porous network and had a rough surface due to the $\beta$-TCP nanoparticles, which were distributed widely and uniformly throughout the gelatin-fiber matrix. The repair efficacy of rabbit mandible defects implanted with bone substitute (Bio-Oss) and covered with the gelatin/ $\beta$-TCP composite nanofibrous membrane was evaluated in comparison with pure gelatin nanofibrous membrane. Gross observation, histological examination, and immunohistochemical analysis showed that new bone formation and defect closure were significantly enhanced by the composite membranes compared to the pure gelatin ones. From these results, we conclude that nanofibrous gelatin/ $\beta$-TCP composite membranes could serve as effective barrier membranes for guided tissue regeneration.
\end{abstract}

\section{Introduction}

Precisely designed scaffolds with controlled structural organization and desirable biochemical properties play a central role in tissue engineering $[1,2]$. When used in regenerative medicine, the goal is often to mimic natural tissue microenvironments by manufacturing scaffolds with similar biological, chemical, and mechanical properties [3, 4]. The electrospinning technique has been gaining attention in regenerative medicine because of the resulting ultrafine fibers, which physically mimic natural bone extracellular matrix (ECM) at the nanoscale [5-9]. Moreover, the surface morphology, architecture, and performance of the fibrous matrix can be controlled by modifying the composition or proportions of its components [10-13]. The simple and relatively inexpensive setup (a glass syringe pump, high-voltage generator, and metallic collector) also accounts for its wide application in generating nanofibers from a wealth of natural and synthetic organic polymers $[14,15]$.

Gelatin is a protein biopolymer that is derived from the partial hydrolysis of natural collagen. It is widely used as a scaffold material for tissue engineering because of its availability, low cost, excellent functional properties, and, in particular, its lack of antigenicity [16-18]. Additionally, it is considered to provide integrin binding sites for cell adhesion [19]. In bone tissue engineering studies, hydroxyapatite (HA), $\beta$-tricalcium phosphate ( $\beta$-TCP), or other bioactive ceramics are commonly added to the gelatin matrix to improve 
the osteoinductivity [20-22]. Recently, the potential application of gelatin/HA composites in bone tissue regeneration has been extensively studied $[23,24]$. The composite materials significantly improved the biological properties in comparison with the pure gelatin equivalent. Although HA has been found to possess good biocompatibility and osteoinductivity, it is insufficiently biodegradable $[25,26]$. $\mathrm{Ca}^{2+}$ ions in the local environment are known to enhance the proliferation and differentiation of osteoblasts by membranemediated ionic transfer $[21,27]$. Thus, the biodegradability of $\beta$-TCP makes it an excellent candidate for the repair of bone defects because it dissolves in vivo $[28,29] . \mathrm{Ca}^{2+}$ ions released from $\beta$-TCP can bind to the carboxyl groups of the gelatin molecular chain, leading to ionic interactions that promote the differentiation of osteoblasts [30].

It is well known that cells have an inherent ability to detect and respond to the surface properties of their substrate $[31,32]$. The efficacy of gelatin/ $\beta$-TCP composite scaffolds in the guidance of growth, function, and organization of boneforming cells has been recently demonstrated $[22,33,34]$. In our previous study [35], different proportions of $\beta$-TCP nanoparticles were successfully incorporated into electrospun gelatin nanofibers to obtain heterojunctional composite nanofibers. Based on in vitro results, it was suggested that osteoblast attachment, spreading, proliferation, and differentiation were accelerated in proportion to the content of $\beta$ TCP nanoparticles and the resulting release of $\mathrm{Ca}^{2+}$ into the medium. However, the efficacy of these composite nanofibers in promoting in situ bone defect repair has not yet been explored.

In the present study, pure gelatin nanofibers and gelatin/ $\beta$-TCP composite nanofibers with $20 \mathrm{wt} . \% \beta$-TCP loading were fabricated by electrospinning. The surface morphology of these nanofibers was characterized via scanning electron microscopy (SEM). The therapeutic effectiveness of gelatin/ $\beta$-TCP composite nanofibers for repair of criticalsized bone defects in the rabbit mandible was examined. At 4 and 12 weeks after implantation, histological and immunohistochemical analyses were combined with gross observation to assess new bone formation.

\section{Materials and Methods}

2.1. Materials. Gelatin ( $\mathrm{pH} 4.5-5.5$, Bloom Number 240270) was purchased from Amresco Chemical Company (Solon, OH, USA). $\beta$-TCP nanoparticles (average particle size $\sim 200 \mathrm{~nm}$ ) were supplied by Rebone Biomaterials Company (Shanghai, China). 1-Ethyl-3-(3-dimethyl aminopropyl) carbodiimide $\mathrm{HCl}$ (EDC-HCl; Sigma-Aldrich, St. Louis, MO, USA), N-hydroxy-succinimide (NHS; Sigma-Aldrich), and sodium citrate (Amresco) were used in this study. The commercially available Bio-Oss bone substitute was supplied by Geistlich-Pharma (Wolhusen, Switzerland).

2.2. Preparation of Spinning Solution. Aqueous solutions of gelatin were prepared by dissolving gelatin in deionized water under gentle stirring at $40^{\circ} \mathrm{C}$ for $3 \mathrm{~h}$. A defined amount of $\beta$-TCP nanoparticles was suspended by ultrasonication and vigorous stirring in water containing $2 \% \mathrm{w} / \mathrm{v}$ sodium citrate, before the addition of polymers. At a fixed gelatin concentration $(20 \% \mathrm{w} / \mathrm{v})$, the $\beta$-TCP content was set as $20 \%$ of the gelatin.

2.3. Preparation of Electrospun Nanofibers. The detailed procedure for the electrospinning of the aqueous gelatin solution is described in our previous work [35]. Briefly, electrospinning was carried out in an apparatus equipped with a heating device. The environmental temperature of the electrospinning process was maintained at $40^{\circ} \mathrm{C}$. The aforementioned gelatin and gelatin $/ \beta$-TCP solutions were electrospun at a high voltage of $20 \mathrm{kV}$ (electrospinning apparatus; Beijing Machinery \& Electricity Institute, Beijing, China). The syringe was connected to a stainless-steel needle (ID $=1.6 \mathrm{~mm}$ ). A feeding rate of $0.3 \mathrm{~mL} / \mathrm{h}$ was set using a syringe pump (TOP 5300, Tokyo, Japan). The distance between the spinneret and the grounded aluminum collector was fixed at $12 \mathrm{~cm}$. The membranes were collected on the surface of the aluminum foil and dried at room temperature in a vacuum for $12 \mathrm{~h}$.

2.4. Crosslinking of Electrospun Nanofibers. The electrospun membranes were chemically crosslinked in accordance with our previous work [36]. Briefly, four sheets of electrospun fibrous membranes (thickness, $\sim 100 \mu \mathrm{m}$ ) were laminated and immersed in $200 \mathrm{~mL}$ ethanol/water (volume ratio, 9:1) solution containing EDC/NHS (molar ratio, $2.5: 1$ ) for $24 \mathrm{~h}$ at $4^{\circ} \mathrm{C}$. After crosslinking, unbound and excess EDC and NHS were removed by rinsing the membranes thoroughly with $100 \mathrm{mM}$ phosphate-buffered saline (PBS). This was followed by repeated washing with distilled water. The membrane was frozen at $-70^{\circ} \mathrm{C}$ for $3 \mathrm{~h}$ and then freeze-dried overnight under vacuum.

2.5. Characterization of Gelatin/ $\beta$-TCP Composite Nanofibers. The surface morphology and internal structure of the composite nanofibers were observed via scanning electron microscopy (SEM; S-4700 microscope; Hitachi, Tokyo, Japan). The distribution of $\beta$-TCP nanoparticles in the gelatin nanofiber matrix was investigated by transmission electron microscopy (TEM; H-800 microscope; Hitachi).

2.6. Animals and Surgical Procedures. A total of 18 male rabbits (New Zealand White Rabbits, about $3.0 \mathrm{~kg}$ of weight) were used in this study. The experimental protocol was approved by the Animal Care and Use Committee of Peking University. Rabbits were divided randomly into three groups: (1) 12 defects for implantation of Bio-Oss granules and coverage with gelatin/ $\beta$-TCP composite membranes, (2) 12 defects for implantation of Bio-Oss granules and coverage with pure gelatin membranes, and (3) 12 defects left untreated (no implant or membrane). The rabbits were anesthetized with sodium pentobarbital and a $3 \mathrm{~cm}$ parallel incision was made along the inferior border of the mandible on both sides. The periosteum was then retracted to expose the submaxilla. Circular critical-size defects, $8-\mathrm{mm}$ in diameter, were made in the buccal-lingual direction on one side of the mandible of each rabbit (Figure 2). In the treated groups, $0.25 \mathrm{~g}$ of 
Bio-Oss granules was implanted into the defect and covered with a pure gelatin membrane or a gelatin/ $\beta$-TCP composite membrane. The mucoperiosteal flaps were then carefully sutured over the defect areas of all the rabbits (groups 1-3) using 3-0 absorbable sutures. The rabbits were sacrificed at 4 and 8 weeks after implantation for tissue processing.

2.7. Gross Observation. At 4 and 12 weeks after implantation, intact mandibles were harvested and fixed in $4 \%$ paraformaldehyde for $24 \mathrm{~h}$ at $4^{\circ} \mathrm{C}$, and the mandible defect site was assessed by gross observation.

2.8. Histological Analysis. Tissue processing and sectioning were carried out as previously described [37]. Briefly, tissue samples were fixed in 10\% neutral buffered formalin for 7 days, decalcified and dehydrated according to standard protocols, embedded in paraffin, and sectioned at a thickness of $5 \mu \mathrm{m}$. Masson's trichrome staining was performed separately on tissue sections, according to the manufacturer's instructions, and images were captured using a light microscope (CX21, Olympus, Japan). After staining, each section was observed under light microscopy at 100x magnification and at least 10 images were randomly captured for each section. Using image analysis software, Image-ProPlus (Media Cybernetics, USA), the bone area was expressed as a percentage (\%) of the measured total tissue area in each group.

2.9. Immunohistochemical Analysis. Immunostaining for osteocalcin (OCN) was performed as we have described previously [38]. Briefly, tissue slides were deparaffinized and rehydrated and then submerged in hydrogen peroxide to quench peroxidase activity. Before exposure to the primary antibody against OCN (ab13420; CA 1:100; Abcam, USA), slides were incubated with $1 \%$ BSA to block nonspecific binding. After overnight incubation with the primary antibodies at $4^{\circ} \mathrm{C}$, HRP conjugated secondary antibodies were applied to the slides for 1 hour at room temperature. Finally, a diaminobenzidine (DAB) kit (Beyotime, Jiangsu, China) was used to develop the color, followed by counterstaining with hematoxylin. Slides were observed under a light microscope (CX21, Olympus, Japan). OCN expression within the defect area was quantified using a publicly available web application, ImunoRatio $[39,40]$.

2.10. Statistical Analysis. Quantitative data were presented as mean \pm standard deviation (SD). Statistical differences between groups were evaluated via a Student's $t$-test using the software package, SPSS 13.0 (SPSS Science). Differences between groups were considered statistically significant if $P<$ 0.05 and highly significant if $P<0.01$.

\section{Results and Discussion}

3.1. Morphology of Electrospun Gelatin/ $\beta$-TCP Composite Nanofibers. Gelatin is temperature-sensitive, so the electrospinning process was performed at $40^{\circ} \mathrm{C}$ to avoid gel formation. Prior to crosslinking, all the electrospun nanofibers showed a nonwoven structure with an interconnected porous network (Figure 1). The diameters of the nanofibers appeared approximately uniform under SEM. Pure gelatin nanofibers were continuous, smooth, and homogeneous (Figure 1(a)). Composite nanofibers had a rough surface because of the incorporation of $\beta$-TCP nanoparticles (Figure 1(b)). It has been reported that the rough nanofiber surface created by apatite particles can promote cell adhesion, proliferation, and osteogenic differentiation of bone-forming cells [41]. TEM (subinset of Figure 1(b)) showed that the $\beta$-TCP nanoparticles were embedded in the nanofibers. After crosslinking, the fibers became curled and conglutinated. In addition, the pore size decreased substantially and there was a clear increase in fiber diameter due to swelling induced by the crosslinking procedure. In our previous study, electrospun gelatin/ $\beta$-TCP composite nanofibers with $20 \mathrm{wt} . \% \beta$-TCP possessed most remarkable biological properties in terms of osteoblasts attachment, cell spreading, cell proliferation, and early osteogenic differentiation [35]. In this study, the ability of these composite nanofibers to promote and guide bone regeneration in situ was investigated.

3.2. Gross Observation of Mandible Defect Repair. Figure 3 shows macroscopic images used to evaluate rabbit mandible defects that were implanted for 4 or 12 weeks. At 4 weeks after operation, the implants were completely filled with nascent bone, but the boundary between nascent and host bone could be seen distinctly (Figures 3(b) and 3(c)). More specifically, nascent bone in the defects implanted with a composite membrane appeared denser than that in the pure gelatin group. The defect exhibited no signs of recovery in the untreated group and conspicuous introcession remained after the filled fibrous tissue was detached (Figure 3(d)).

At 12 weeks after implantation (Figure 3(f)), the defects treated using the composite membranes were well healed and the nascent bone was indistinguishable from the host bone. On the other hand, for pure gelatin membranes at this time point, the nascent bone was slightly less dense than the surrounding host bone (Figure 3(e)). In the untreated group, obvious introcessions remained at the defect sites (Figure 3(d)) demonstrating the critical size of the defects. These results indicate that the composite membrane increased the rate of bone repair via the contribution of $\beta$ TCP. They are consistent with the results of another study, which found that the addition of apatite into electrospun poly(lactide-co-caprolactone) (PLCL) nanofibers improved biocompatibility and bone formation [42].

3.3. Histological Assessment of Mandible Defect Repair. The newly formed tissue within the mandible defect was further analyzed by histological staining, as shown in Figure 4. At 4 weeks after implantation, the boundary between the defect area and the host bone tissue could still be clearly identified (marked by black arrows). Masson staining showed that the fibrous tissue surrounding the implants was mainly composed of newly formed and regularly aligned collagen fibers that were stained green (Figures 4(b) and 4(c)). Specifically, implanted defects that used a composite membrane contained more green-stained immature nascent bone than those with pure gelatin membranes. Interestingly, nascent bone could close over and grow into the residual 


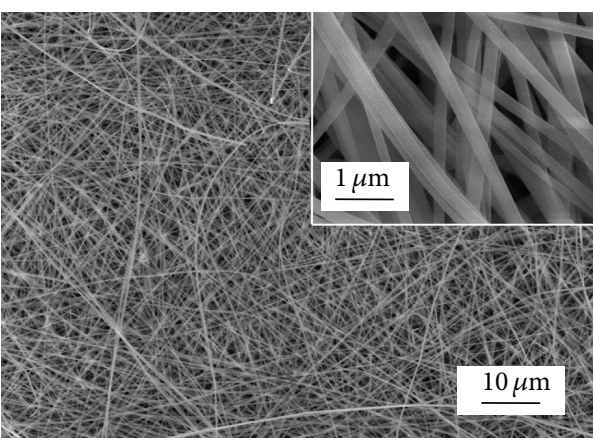

(a)

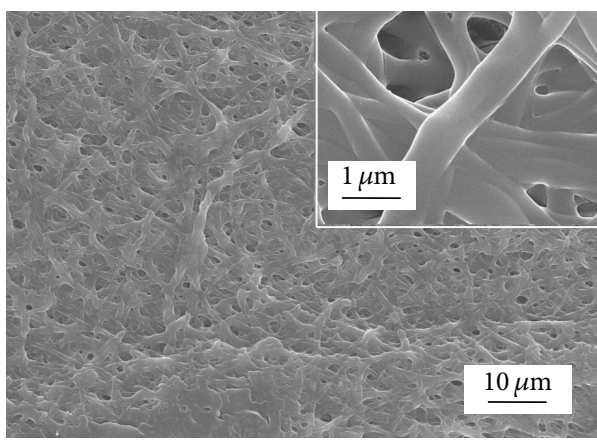

(c)

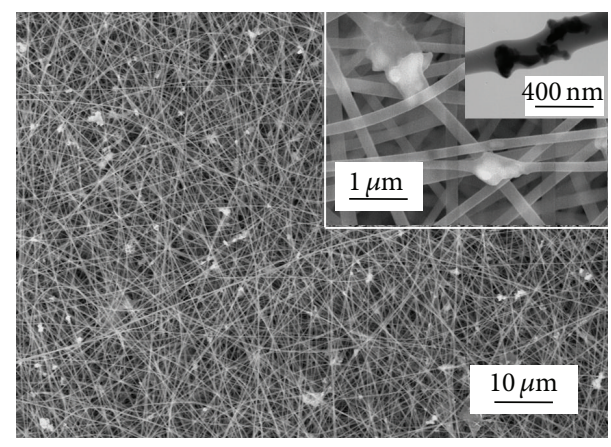

(b)

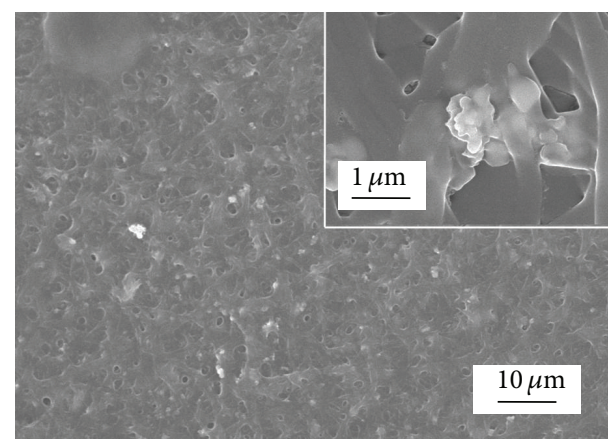

(d)

FIGURE 1: Scanning electron micrographs of electrospun nanofibers before ((a), (b)) and after ((c), (d)) crosslinking; gelatin nanofibers ((a), (c)); gelatin/ $\beta$-TCP composite nanofibers ((b), (d)). The inset shows an enlarged view. A transmission electron micrograph of gelatin/ $\beta$-TCP composite nanofibers is shown in the subinset of (b).

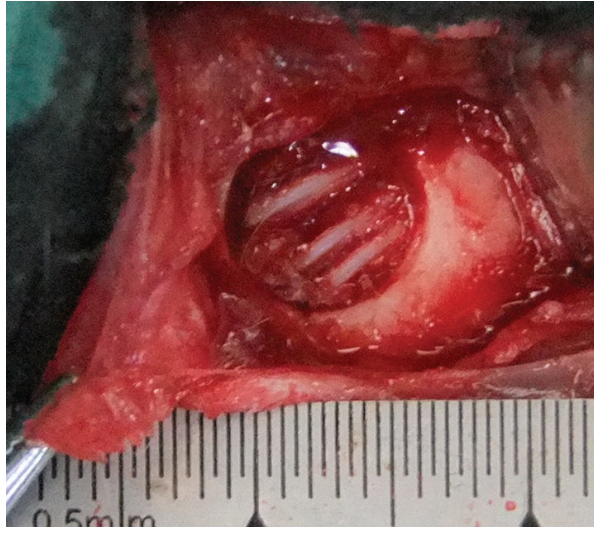

(a)

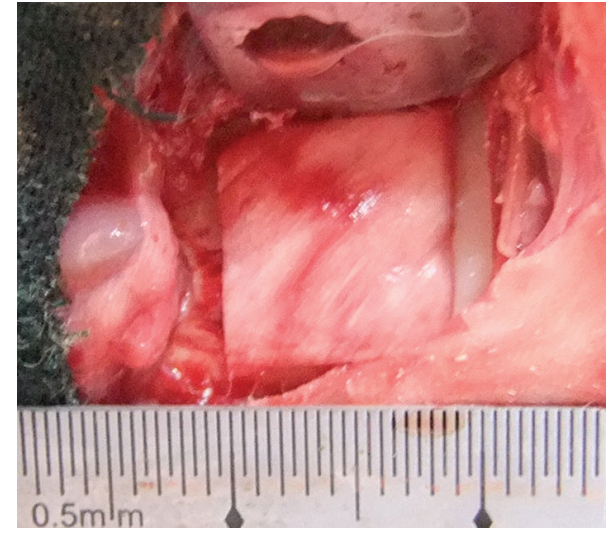

(b)

FIGURE 2: Illustration of the surgical procedure. (a) Bone defects of 8-mm in diameter created in the region anterior to the jaw angles of the rabbit mandible. (b) Defect filled with Bio-Oss granules and covered with gelatin or gelatin/ $\beta$-TCP composite membrane.

implanted Bio-Oss scaffolds (marked by yellow arrow). These results imply that the composite membrane is an excellent barrier for creation of a microenvironment suitable for bone regeneration. In contrast, numerous fat vacuoles at the untreated defect sites further demonstrated the poor healing that occurred in the absence of treatment (Figure 4(a)).

The interface between nascent bone and the host bone became indistinguishable in both treatment groups at 12 weeks after operation (Figures 4(e) and 4(f)). Masson staining showed that the green-stained collagen fibers described above were replaced by mature woven bone in the central region of the defect (Figures 4(e) and 4(f)). The use of a composite membrane resulted in the formation of more regular bony trabeculae and more mature-bone-marrow cavities than those seen for the pure gelatin membrane. Again, the untreated defects looked very different, showing 


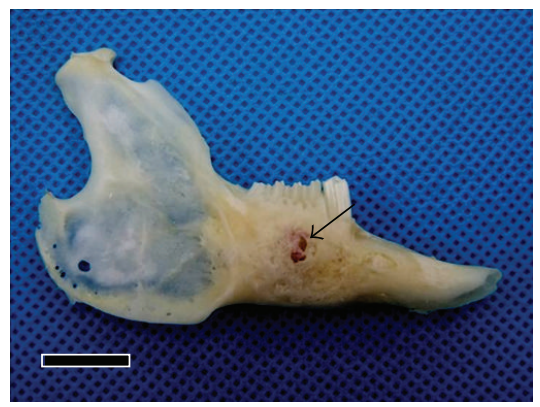

(a)

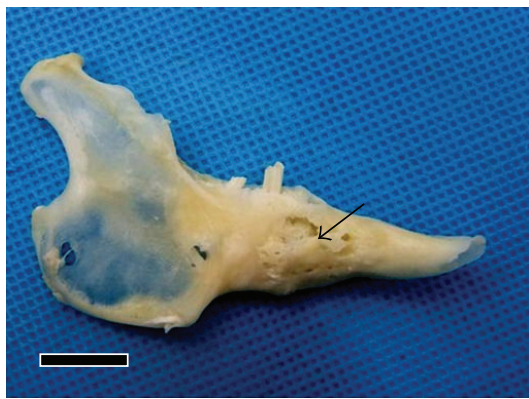

(d)

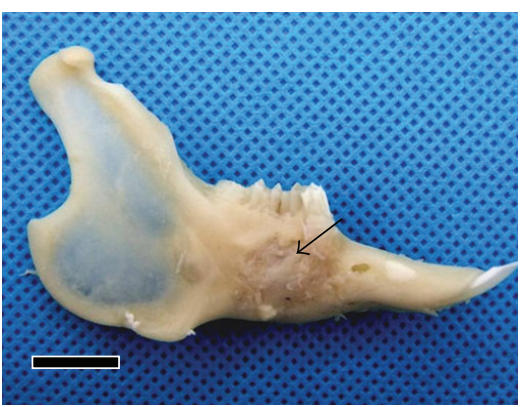

(b)

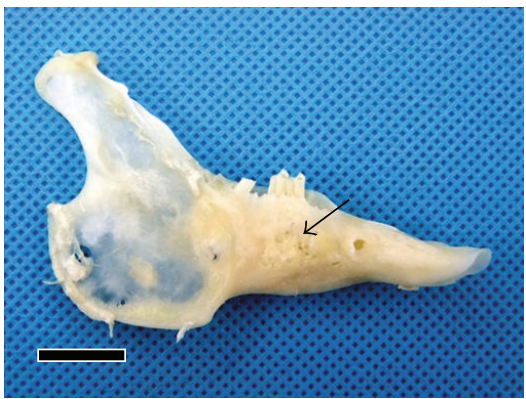

(e)

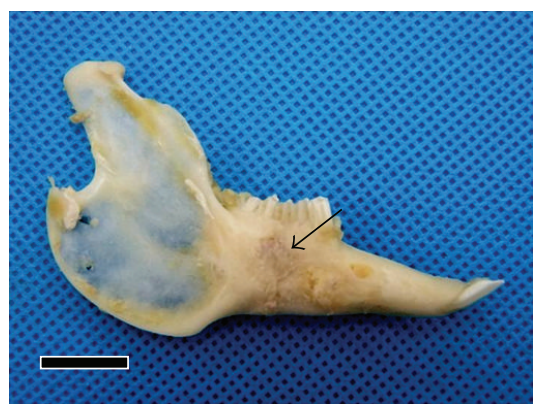

(c)

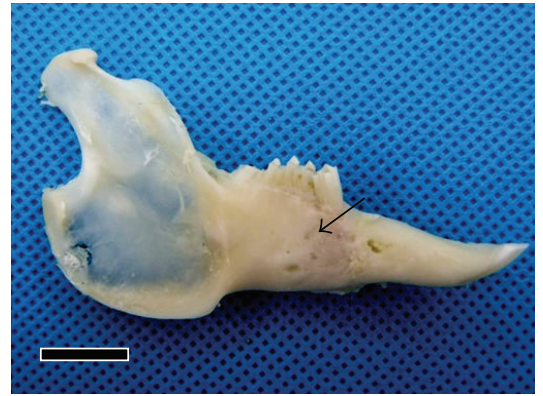

(f)

FIGURE 3: Representative macroscopic photographs of mandible defects at 4 weeks ((a)-(c)) and 12 weeks ((d)-(f)) after implantation: untreated group ((a), (d)), gelatin group ((b), (e)), and gelatin/ $\beta$-TCP group ((c), (f)). Black arrows denote the surgical site. Scale bar: 10 mm.

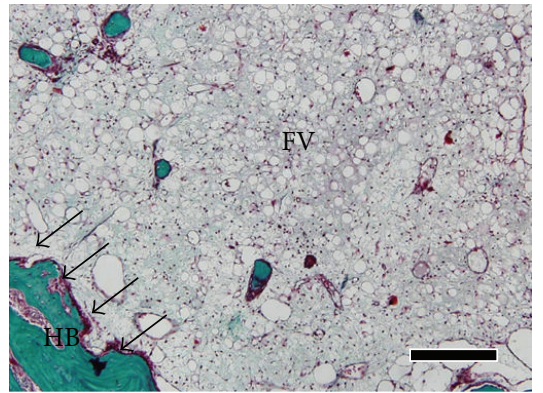

(a)

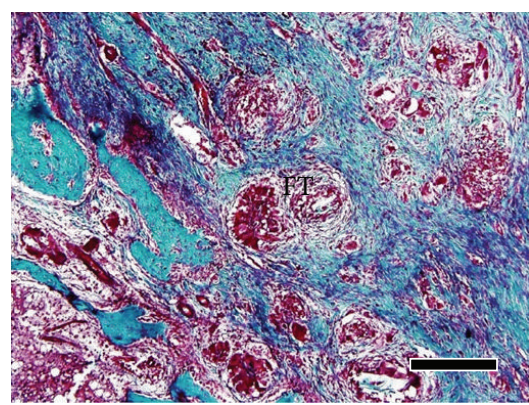

(d)

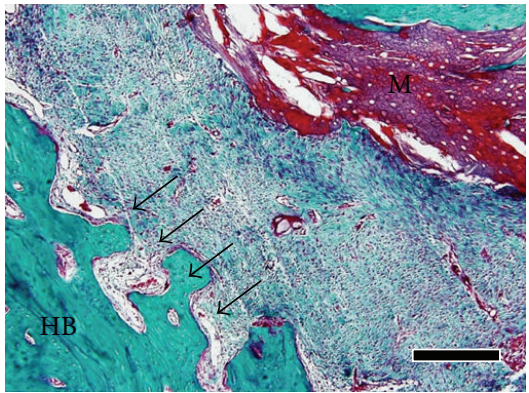

(b)

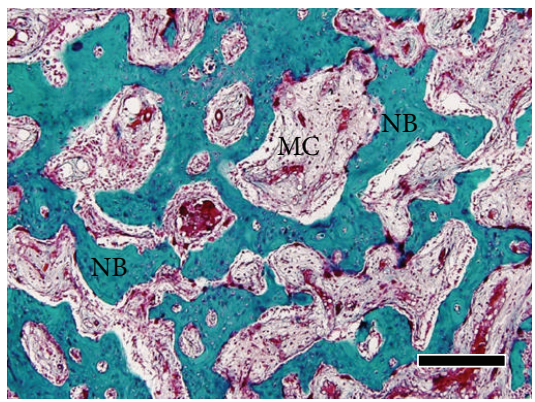

(e)

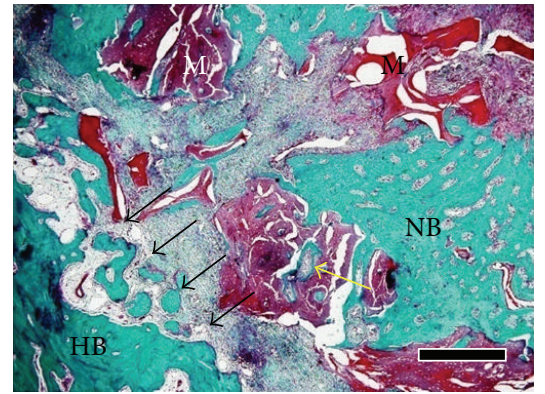

(c)

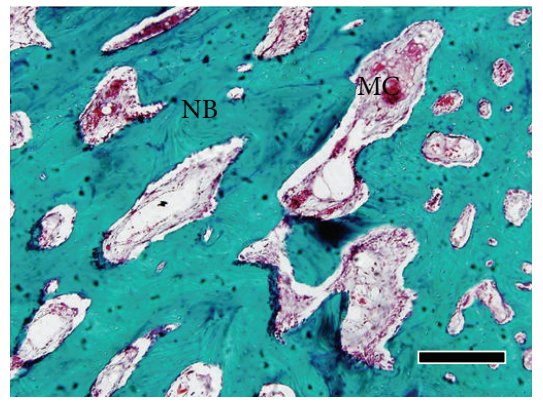

(f)

FIGURE 4: Representative Masson staining micrographs of bone at site of defect after 4 weeks ((a)-(c)) and 12 weeks ((d)-(f)) of implantation: untreated group ((a), (d)), gelatin group ((b), (e)), and gelatin/ $\beta$-TCP group ((c), (f)). Black arrows denote the boundary between nascent bone and host bone. $\mathrm{M}$, material; $\mathrm{HB}$, host bone; NB, nascent bone; MC, medullary cavity; FV, fat vacuoles; and FT, fibrous tissue. Scale bar: $100 \mu \mathrm{m}$. 


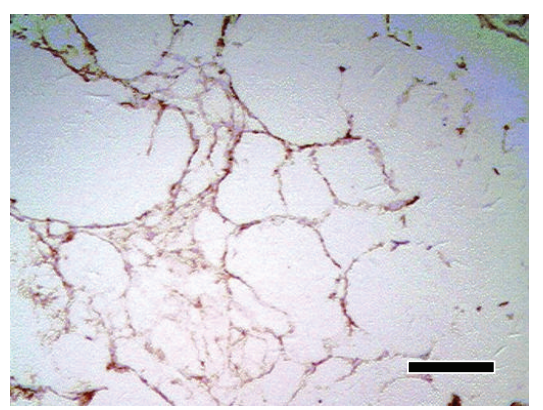

(a)

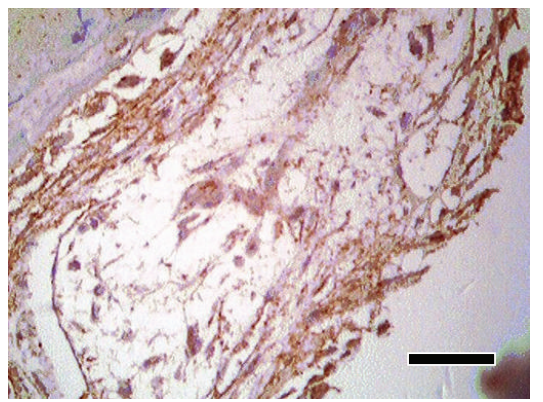

(d)

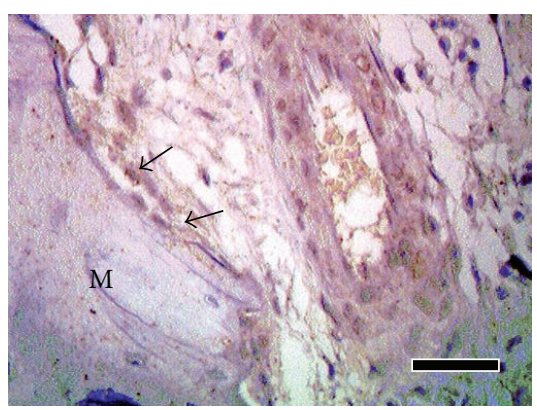

(b)

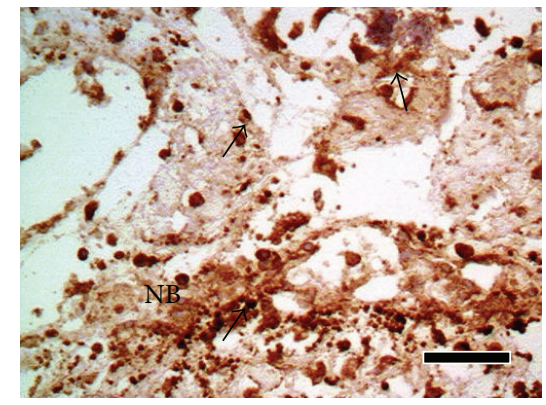

(e)

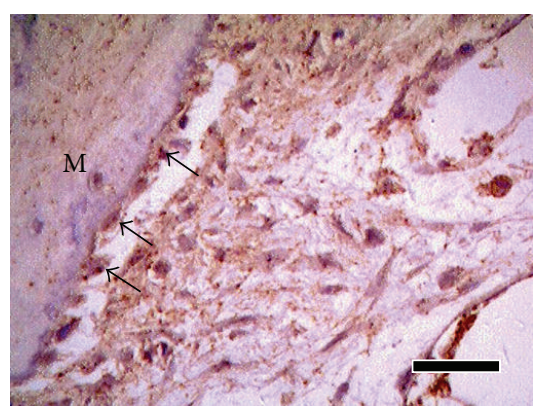

(c)

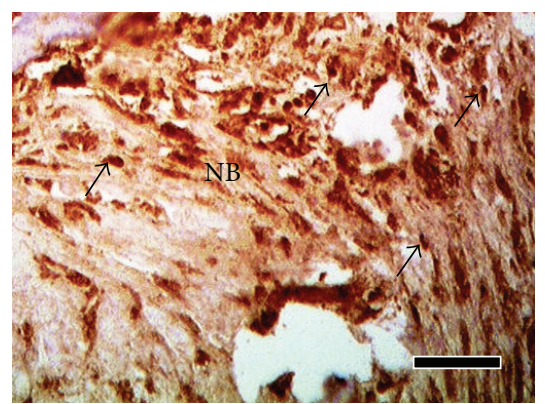

(f)

FIGURE 5: Representative immunostaining micrographs of osteocalcin (OCN) at site of defect after 4 weeks ((a)-(c)) and 12 weeks ((d)-(f)) of implantation: untreated group ((a), (d)), gelatin group ((b), (e)), and gelatin/ $\beta$-TCP group ((c), (f)). The arrows denote positive expression of OCN. M, material and NB, nascent bone; scale bar: $50 \mu \mathrm{m}$.

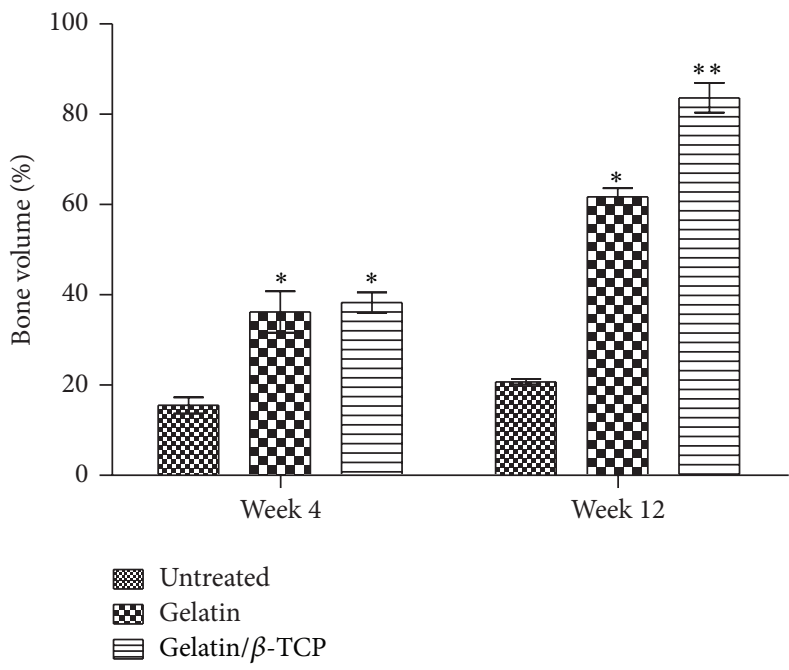

(a)

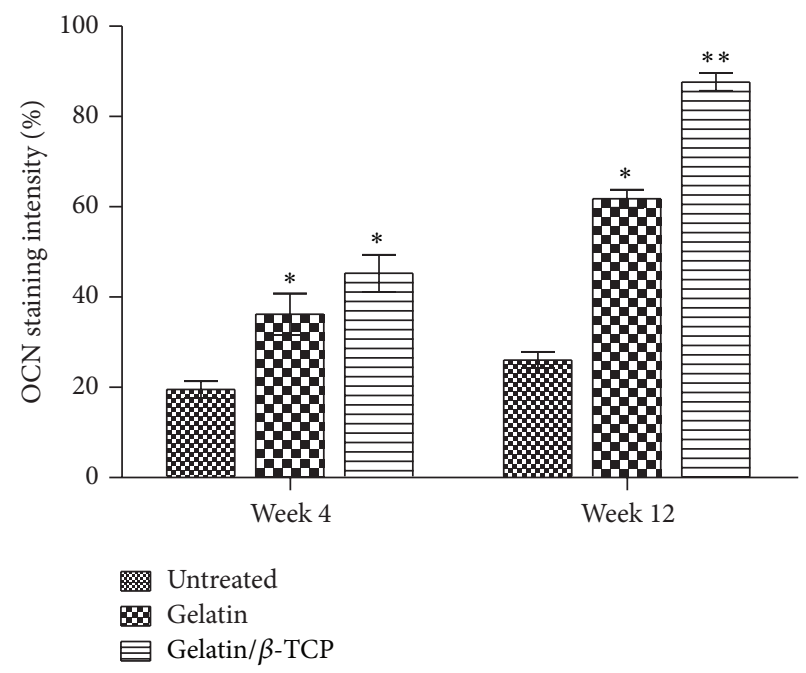

(b)

FIGURE 6: Bone area measurements (a) and osteocalcin staining intensity (b) of rabbit mandible defects. Values represent the mean \pm SD; $n=3,{ }^{*} P<0.05$, and ${ }^{* *} P<0.01$.

numerous red-stained blood clots and small green-stained collagen fibers (Figure 4(d)). As summarized in Figure 6(a), the degree of healing and quality of nascent bone formation were related to treatment as follows: composite membrane $>$ pure gelatin membrane $\gg$ no treatment.
3.4. Immunohistochemical Assessment of Mandible Defect Repair. Osteocalcin (OCN), a major noncollagenous protein component of bone extracellular matrix, is synthesized and secreted exclusively by osteoblasts in the late stage of bone maturation. At 4 weeks after operation, OCN staining was 
prevalent within the defects of both treated groups (Figures 5(b) and 5(c)). At 12 weeks after implantation, the most intense OCN staining was observed in the composite membrane group (Figure 5(f)). Quantitative analysis of OCN protein (Figure 6(b)) corresponded to the levels of nascent bone formation observed via Masson staining. The differences in osteogenesis may be explained by the release of calcium ions from the incorporated $\beta$-TCP nanoparticles. Our previous research confirmed the pivotal role of continuous $\mathrm{Ca}^{2+}$ release from gelatin/ $\beta$-TCP composite nanofibers in regulating the osteoblast response [35]. In others studies, it was also found that optimal calcium-ion concentrations could significantly promote osteogenic differentiation and mineralization of stem cells [43-45]. Overall, these electrospun nanofibrous gelatin/ $\beta$-TCP composite membranes have been shown to exhibit a high degree of biocompatibility and to enhance the effectiveness of bone defect healing. Therefore, they may be beneficial for regeneration of hard tissue, particularly for guided bone regeneration in periodontology.

\section{Conclusions}

In the present study, composite nanofibers made of gelatin/ $\beta$ TCP were produced by electrospinning. The composite nanofibers exhibited a nonwoven structure with an interconnected porous network and had a rough surface due to the incorporated $\beta$-TCP nanoparticles, which were widely and uniformly distributed in the gelatin-fiber matrix. When bone substitute (Bio-Oss) implanted into rabbit mandible defects was covered by a composite membrane, new bone formation and defect closure were significantly enhanced compared to coverage by pure gelatin membranes. In summary, this study demonstrates that the electrospun nanofibrous gelatin/ $\beta$ TCP composite membranes developed here have great potential for use in periodontal therapy as barriers for guided bone regeneration.

\section{Conflict of Interests}

The authors declare that there is no conflict of interests regarding the publication of this paper.

\section{Authors' Contribution}

Mingming $\mathrm{Xu}$ and Xuehui Zhang contributed equally to this paper.

\section{Acknowledgments}

The authors acknowledge funding from the National Basic Research Program of China (2012CB933900), the Key International S\&T Cooperation Project (2011DFA32190), the National Natural Science Foundation of China (81171000, 51302005), the National High Technology Research and Development Program of China (2015AA032004, 2012AA022501), Beijing natural science foundation (7144256, 7144257), Doctoral Scientific Fund Project of the Ministry of Education of China (20130001120112), Beijing Nova Program
(Z14111000180000), and Medical-Engineering Crossover Fund of Peking University (BMU20140406).

\section{References}

[1] M. M. Stevens and J. H. George, "Exploring and engineering the cell surface interface," Science, vol. 310, no. 5751, pp. 1135-1138, 2005.

[2] M. P. Lutolf, P. M. Gilbert, and H. M. Blau, "Designing materials to direct stem-cell fate," Nature, vol. 462, no. 7272, pp. 433-441, 2009.

[3] M. P. Lutolf and J. A. Hubbell, "Synthetic biomaterials as instructive extracellular microenvironments for morphogenesis in tissue engineering," Nature Biotechnology, vol. 23, no. 1, pp. 47-55, 2005.

[4] J. A. Burdick and G. Vunjak-Novakovic, "Engineered microenvironments for controlled stem cell differentiation," Tissue Engineering-Part A, vol. 15, no. 2, pp. 205-219, 2009.

[5] A. Martins, J. V. Araújo, R. L. Reis, and N. M. Neves, "Electrospun nanostructured scaffolds for tissue engineering applications," Nanomedicine, vol. 2, no. 6, pp. 929-942, 2007.

[6] C. Q. Wang, S. Zhang, X. P. Yang, and X. L. Deng, "The effect of alignment of poly (l-lactic acid) (PLLA) nanofibers on osteogenic response of human osteoblast-like MG63 cells," The Journal of the Mechanical Behavior of Biomedical Materials, vol. 4, pp. 600-609, 2011.

[7] N. Yan, X. Zhang, Q. Cai et al., "The effects of lactidyl/glycolidyl ratio and molecular weight of poly(D,L-lactide-co-glycolide) on the tetracycline entrapment and release kinetics of drug-loaded nanofibers," Journal of Biomaterials Science, Polymer Edition, vol. 23, no. 8, pp. 1005-1019, 2012.

[8] X.-L. Deng, G. Sui, M.-L. Zhao, G.-Q. Chen, and X.-P. Yang, "Poly(L-lactic acid)/hydroxyapatite hybrid nanofibrous scaffolds prepared by electrospinning," Journal of Biomaterials Science, Polymer Edition, vol. 18, no. 1, pp. 117-130, 2007.

[9] H. Liu, Q. Cai, P. Lian et al., “The biological properties of carbon nanofibers decorated with $\beta$-tricalcium phosphate nanoparticles," Carbon, vol. 48, no. 8, pp. 2266-2272, 2010.

[10] G. Sui, X. Yang, F. Mei et al., "Poly-L-lactic acid/hydroxyapatite hybrid membrane for bone tissue regeneration," Journal of Biomedical Materials Research-Part A, vol. 82, no. 2, pp. 445454, 2007.

[11] D. Grafahrend, K.-H. Heffels, M. V. Beer et al., "Degradable polyester scaffolds with controlled surface chemistry combining minimal protein adsorption with specific bioactivation," Nature Materials, vol. 10, no. 1, pp. 67-73, 2011.

[12] J. Lee, G. Tae, Y. H. Kim, I. S. Park, S.-H. Kim, and S. H. Kim, "The effect of gelatin incorporation into electrospun poly(llactide-co- $\varepsilon$-caprolactone) fibers on mechanical properties and cytocompatibility," Biomaterials, vol. 29, no. 12, pp. 1872-1879, 2008.

[13] S. Zhang, X. Zhang, Q. Cai, B. Wang, X. Deng, and X. Yang, "Microfibrous $\beta$-TCP/collagen scaffolds mimic woven bone in structure and composition," Biomedical Materials, vol. 5, no. 6, Article ID 065005, 2010.

[14] S. Agarwal, J. H. Wendorff, and A. Greiner, "Progress in the field of electrospinning for tissue engineering applications," Advanced Materials, vol. 21, no. 32-33, pp. 3343-3351, 2009. 
[15] S. A. Sell, P. S. Wolfe, K. Garg, J. M. McCool, I. A. Rodriguez, and G. L. Bowlin, "The use of natural polymers in tissue engineering: a focus on electrospun extracellular matrix analogues," Polymers, vol. 2, no. 4, pp. 522-553, 2010.

[16] K. Morimoto, H. Katsumata, T. Yabuta et al., "Evaluation of gelatin microspheres for nasal and intramuscular administrations of salmon calcitonin," European Journal of Pharmaceutical Sciences, vol. 13, no. 2, pp. 179-185, 2001.

[17] K. Iwanaga, T. Yabuta, M. Kakemi, K. Morimoto, Y. Tabata, and Y. Ikada, "Usefulness of microspheres composed of gelatin with various cross-linking density," Journal of Microencapsulation, vol. 20, no. 6, pp. 767-776, 2003.

[18] S.-M. Lien, L.-Y. Ko, and T.-J. Huang, "Effect of pore size on ECM secretion and cell growth in gelatin scaffold for articular cartilage tissue engineering," Acta Biomaterialia, vol. 5, no. 2, pp. 670-679, 2009.

[19] D. Gupta, J. Venugopal, S. Mitra, V. R. G. Dev, and S. Ramakrishna, "Nanostructured biocomposite substrates by electrospinning and electrospraying for the mineralization of osteoblasts," Biomaterials, vol. 30, no. 11, pp. 2085-2094, 2009.

[20] X. Liu, L. A. Smith, J. Hu, and P. X. Ma, "Biomimetic nanofibrous gelatin/apatite composite scaffolds for bone tissue engineering," Biomaterials, vol. 30, no. 12, pp. 2252-2258, 2009.

[21] H.-W. Kim, H.-E. Kim, and V. Salih, "Stimulation of osteoblast responses to biomimetic nanocomposites of gelatinhydroxyapatite for tissue engineering scaffolds," Biomaterials, vol. 26, no. 25, pp. 5221-5230, 2005.

[22] Y. Takahashi, M. Yamamoto, and Y. Tabata, "Osteogenic differentiation of mesenchymal stem cells in biodegradable sponges composed of gelatin and $\beta$-tricalcium phosphate," Biomaterials, vol. 26, no. 17, pp. 3587-3596, 2005.

[23] H.-W. Kim, J. C. Knowles, and H.-E. Kim, "Porous scaffolds of gelatin-hydroxyapatite nanocomposites obtained by biomimetic approach: characterization and antibiotic drug release," Journal of Biomedical Materials Research-Part B. Applied Biomaterials, vol. 74, no. 2, pp. 686-698, 2005.

[24] M. C. Chang, C.-C. Ko, and W. H. Douglas, "Preparation of hydroxyapatite-gelatin nanocomposite," Biomaterials, vol. 24, no. 17, pp. 2853-2862, 2003.

[25] R. Z. LeGeros, "Properties of osteoconductive biomaterials: calcium phosphates," Clinical Orthopaedics and Related Research, no. 395, pp. 81-98, 2002.

[26] K. M. Woo, J. Seo, R. Zhang, and P. X. Ma, "Suppression of apoptosis by enhanced protein adsorption on polymer/hydroxyapatite composite scaffolds," Biomaterials, vol. 28, no. 16, pp. 2622-2630, 2007.

[27] M. M. Dvorak and D. Riccardi, " $\mathrm{Ca}^{2+}$ as an extracellular signal in bone," Cell Calcium, vol. 35, no. 3, pp. 249-255, 2004.

[28] D. Henrich, C. Seebach, C. Kaehling et al., "Simultaneous cultivation of human endothelial-like differentiated precursor cells and human marrow stromal cells on $\beta$-tricalcium phosphate," Tissue Engineering Part C: Methods, vol. 15, no. 4, pp. 551-560, 2009.

[29] P. Kasten, I. Beyen, P. Niemeyer, R. Luginbühl, M. Bohner, and W. Richter, "Porosity and pore size of $\beta$-tricalcium phosphate scaffold can influence protein production and osteogenic differentiation of human mesenchymal stem cells: an in vitro and in vivo study," Acta Biomaterialia, vol. 4, no. 6, pp. 1904-1915, 2008.
[30] R. J. Jakobsen, L. L. Brown, T. B. Hutson, D. J. Fink, and A. Veis, "Intermolecular interactions in collagen self-assembly as revealed by Fourier transform infrared spectroscopy," Science, vol. 220, no. 4603, pp. 1288-1290, 1983.

[31] P. Habibovic, H. Yuan, C. M. vn Der Valk, G. Meijer, C. A. van Blitterswijk, and K. de Groot, "3D microenvironment as essential element for osteoinduction by biomaterials," Biomaterials, vol. 26, no. 17, pp. 3565-3575, 2005.

[32] N. M. Alves, I. Pashkuleva, R. L. Reis, and J. F. Mano, "Controlling cell behavior through the design of polymer surfaces," Small, vol. 6, no. 20, pp. 2208-2220, 2010.

[33] Y. Takahashi, M. Yamamoto, and Y. Tabata, "Enhanced osteoinduction by controlled release of bone morphogenetic protein2 from biodegradable sponge composed of gelatin and $\beta$ tricalcium phosphate," Biomaterials, vol. 26, no. 23, pp. 48564865, 2005.

[34] Y. Yin, F. Ye, J. Cui, F. Zhang, X. Li, and K. Yao, "Preparation and characterization of macroporous chitosan-gelatin/ $\beta$-tricalcium phosphate composite scaffolds for bone tissue engineering," Journal of Biomedical Materials Research Part A, vol. 67, no. 3, pp. 844-855, 2003.

[35] X. Zhang, Q. Cai, H. Liu et al., "Calcium ion release and osteoblastic behavior of gelatin/beta-tricalcium phosphate composite nanofibers fabricated by electrospinning," Materials Letters, vol. 73, pp. 172-175, 2012.

[36] S. Zhang, Y. Huang, X. Yang et al., "Gelatin nanofibrous membrane fabricated by electrospinning of aqueous gelatin solution for guided tissue regeneration," Journal of Biomedical Materials Research Part A, vol. 90, no. 3, pp. 671-679, 2009.

[37] X. Zhang, Q. Cai, H. Liu et al., "Osteoconductive effectiveness of bone graft derived from antler cancellous bone: an experimental study in the rabbit mandible defect model," International Journal of Oral and Maxillofacial Surgery, vol. 41, no. 11, pp. 1330-1337, 2012.

[38] X. Zhang, M. Xu, L. Song et al., "Effects of compatibility of deproteinized antler cancellous bone with various bioactive factors on their osteogenic potential," Biomaterials, vol. 34, no. 36, pp. 9103-9114, 2013.

[39] T. Luo, C. Wu, and Y. Zhang, "The in vivo osteogenesis of Mg or Zr-modified silicate-based bioceramic spheres," Journal of Biomedical Materials Research Part A, vol. 100, no. 9, pp. 22692277, 2012.

[40] V. J. Tuominen, S. Ruotoistenmäki, A. Viitanen, M. Jumppanen, and J. Isola, "ImmunoRatio: a publicly available web application for quantitative image analysis of estrogen receptor (ER), progesterone receptor (PR), and Ki-67," Breast Cancer Research, vol. 12, no. 4, article R56, 2010.

[41] I. O. Smith, X. H. Liu, L. A. Smith, and P. X. Ma, "Nanostructured polymer scaffolds for tissue engineering and regenerative medicine," Wiley Interdisciplinary Reviews: Nanomedicine and Nanobiotechnology, vol. 1, no. 2, pp. 226-236, 2009.

[42] S.-H. Jegal, J.-H. Park, J.-H. Kim et al., "Functional composite nanofibers of poly(lactide-co-caprolactone) containing gelatinapatite bone mimetic precipitate for bone regeneration," Acta Biomaterialia, vol. 7, no. 4, pp. 1609-1617, 2011.

[43] S. D. McCullen, J. Zhan, M. L. Onorato, S. H. Bernacki, and E. G. Loboa, "Effect of varied ionic calcium on human adiposederived stem cell mineralization," Tissue Engineering Part A, vol. 16, no. 6, pp. 1971-1981, 2010. 
[44] S. Maeno, Y. Niki, H. Matsumoto et al., "The effect of calcium ion concentration on osteoblast viability, proliferation and differentiation in monolayer and 3D culture," Biomaterials, vol. 26, no. 23, pp. 4847-4855, 2005.

[45] S. Nakamura, T. Matsumoto, J.-I. Sasaki et al., "Effect of calcium ion concentrations on osteogenic differentiation and hematopoietic stem cell niche-related protein expression in osteoblasts," Tissue Engineering Part A, vol. 16, no. 8, pp. 2467$2473,2010$. 

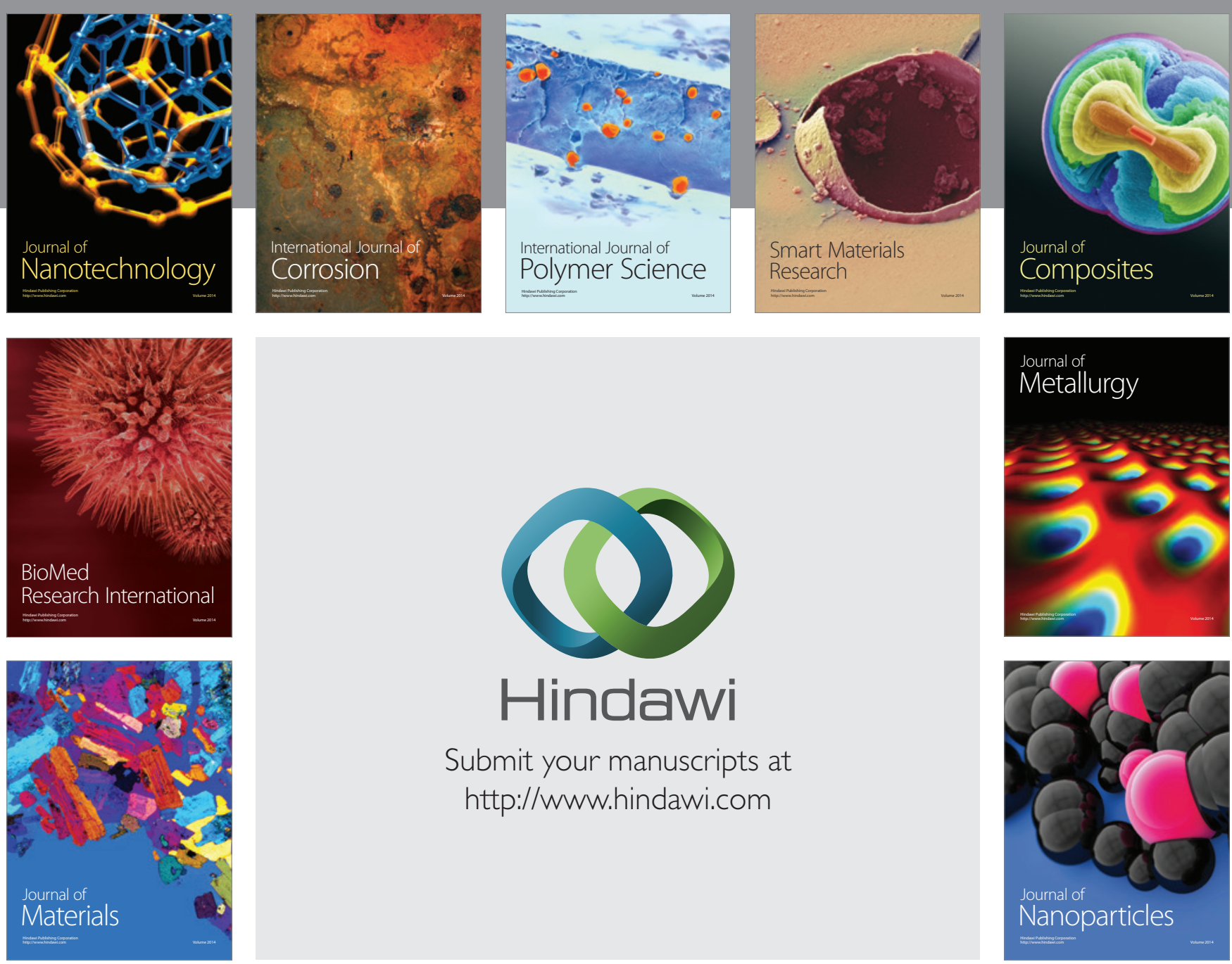

Submit your manuscripts at http://www.hindawi.com
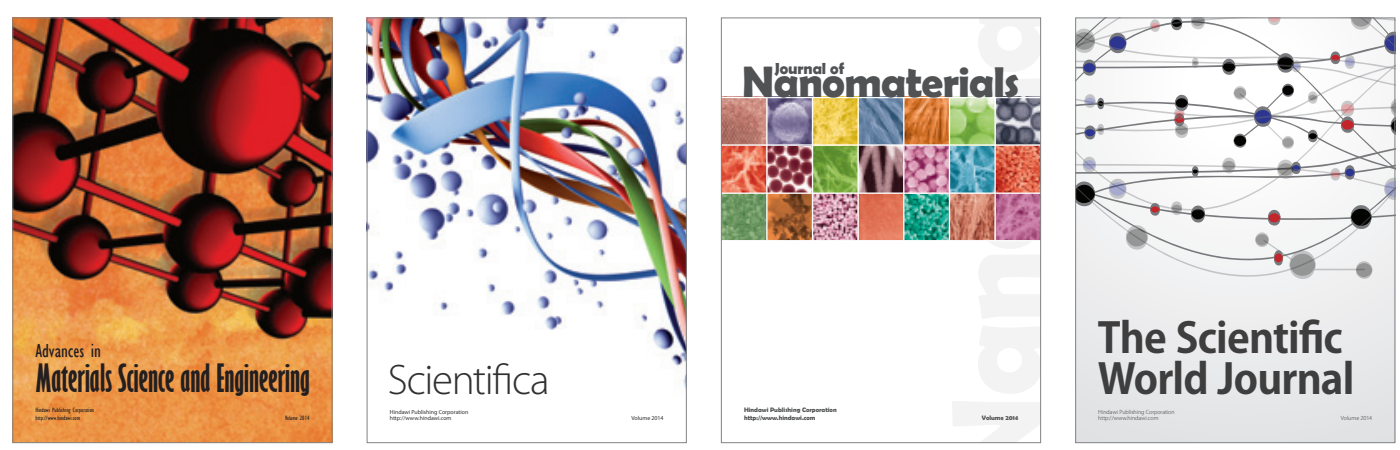

\section{The Scientific World Journal}
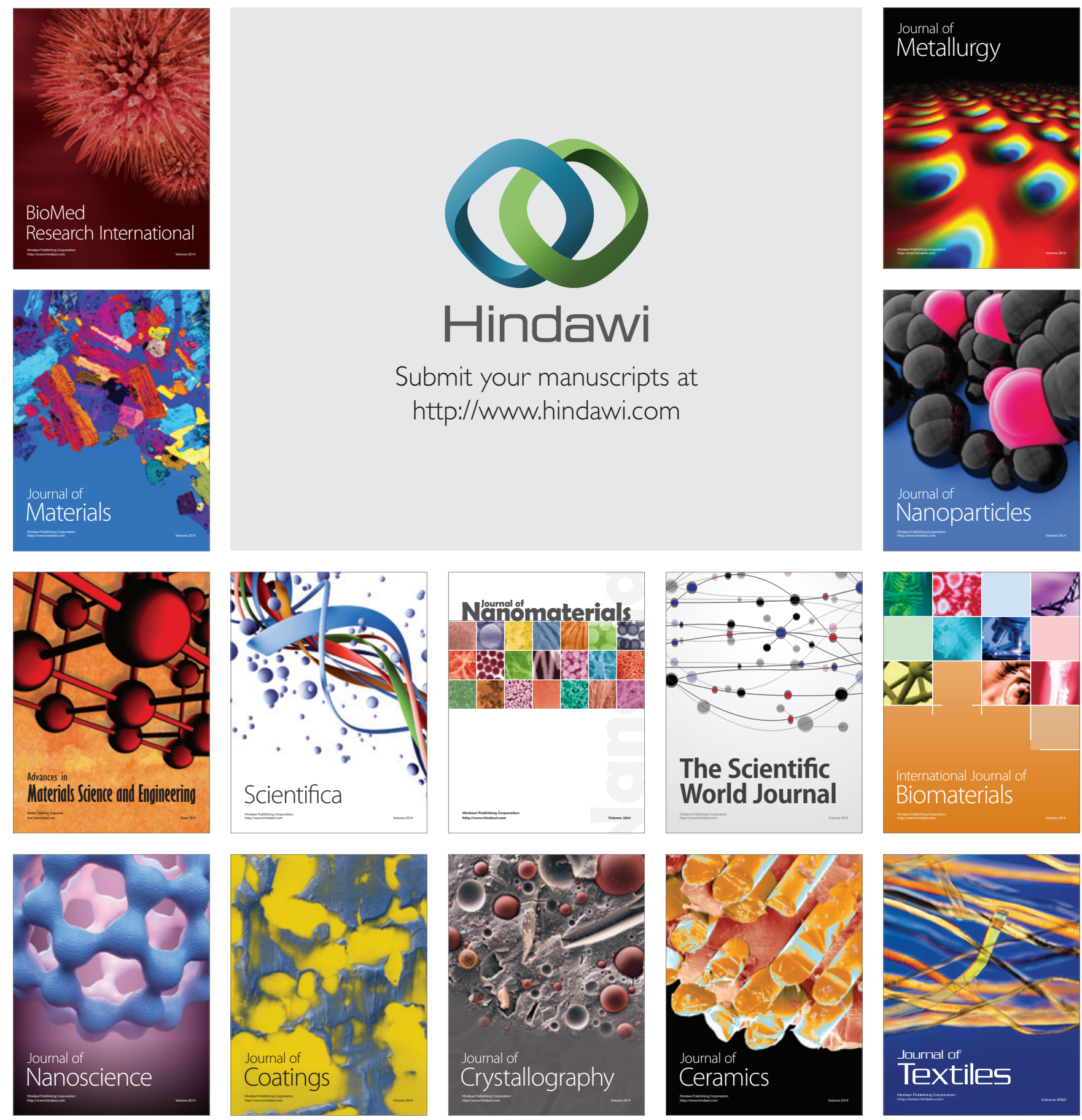